Questions vives

\section{Questions Vives}

Recherches en éducation

$n^{\circ} 21 \mid 2014$

Le travail collectif des enseignants en question(s)

\title{
Garant, M. \& Letor, C., Encadrement et leadership. Nouvelles pratiques en éducation et formation
}

Bruxelles, éditions De Boeck

\section{Baby Lenhard}

\section{CpenEdition}

\section{Journals}

Édition électronique

URL : http://journals.openedition.org/questionsvives/1496

DOI : 10.4000/questionsvives.1496

ISSN : $1775-433 X$

Éditeur

Université Aix-Marseille (AMU)

Édition imprimée

Date de publication : 15 septembre 2014

ISBN : 978-2-912643-45-2

ISSN : 1635-4079

Référence électronique

Baby Lenhard, « Garant, M. \& Letor, C., Encadrement et leadership. Nouvelles pratiques en éducation et formation », Questions Vives [En ligne], n 21 | 2014, mis en ligne le 15 septembre 2014, consulté le 22 septembre 2020. URL : http://journals.openedition.org/questionsvives/1496; DOI : https://doi.org/ 10.4000/questionsvives.1496

Ce document a été généré automatiquement le 22 septembre 2020.

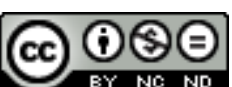

Questions Vives est mis à disposition selon les termes de la licence Creative Commons Attribution Pas d'Utilisation Commerciale - Pas de Modification 4.0 International. 


\section{Garant, M. \& Letor, C., Encadrement et leadership. Nouvelles pratiques en éducation et formation}

Bruxelles, éditions De Boeck

Baby Lenhard

\section{RÉFÉRENCE}

Garant, M., \& Letor, C. (2014). Encadrement et leadership. Nouvelles pratiques en éducation et formation. Bruxelles : De Boeck.

Encadrement et leadership repose sur un constat : l'apparition des nouvelles fonctions de leadership et d'encadrement au sein des organisations scolaires et socio-éducatives. Les auteurs se donnent ainsi pour objectif d'explorer cet espace reconfiguré, produit des mouvements de décentralisation appliqués aux politiques scolaires que connaissent les pays occidentaux, dont une conséquence importante est le transfert d'une partie des pouvoirs du sommet vers la sphère locale. Sous l'impact de l'automisation des structures, la figure traditionnelle du leader explose pour laisser place à une nouvelle catégorie en voie de construction, celle des cadres intermédiaires dont la position d'interface entre la hiérarchie et la base autorise une redistribution des rôles et de nouveaux enjeux de pouvoir. Ainsi, l'ouvrage se propose d'analyser le concept de "leadership distribué » où les missions organisationnelles sont partagées entre différents agents, par opposition au «leadership concentré » qui les centralise, et de mettre à jour les contours et l'implication de ces nouveaux métiers en se focalisant sur les principaux acteurs, pour comprendre de quelle manière ceux-ci parviennent à occuper l'espace intermédiaire émergeant, résultat de nouveaux modes de régulation organisationnels, comment ils le gèrent et l'approprient.

2 L'ouvrage est composé de trois parties et de neuf chapitres, avec un découpage thématique centrant sur différents types d'acteurs: cadres dirigeants (première 
partie), cadres de soutien (deuxième partie) et experts de contrôle et d'accompagnement (troisième partie). Il circonscrit les fonctions d'encadrement et de leadership en analysant l'interaction des acteurs au sein d'une zone d'incertitude nouvellement créée par la décentralisation des systèmes éducatifs en France, en Suisse francophone, en Belgique et au Québec. En s'appuyant également sur les outils de médiation, l'ouvrage décline comment ces acteurs les instrumentent et instrumentalisent pour construire leur action.

3 Le chapitre 1 pose les prémisses du cadre conceptuel général de l'ouvrage : la notion de leadership en tant que construit organisationnel. Ce parti-pris flexibilise le rôle en l'appliquant aussi bien aux responsables hiérarchiques reconnus qu'à tout autre agent s'occupant des missions d'encadrement. Pour Liesbeth Piot et Geert Kelchtermans, le leadership scolaire peut s'assimiler à un processus d'influence sociale visant à fédérer autour d'une certaine vision et les objectifs afférents. La contribution spécifique du chapitre repose sur la proposition d'une approche intégrée du leadership qui dépasse les théories actuelles concurrentes (leadership concentré ou distribué) et insuffisantes (car surestimant ou sous-estimant le rôle de certains acteurs) pour expliquer les réalités du terrain. De cette opposition naît une impasse conceptuelle que les auteurs se proposent de résoudre en prônant l'idée d'un type de leadership moins identifié formellement, issue de l'observation d'une division effective du travail, et qui met l'accent sur le poids des individus, de l'action individuelle et collective situées dans un contexte particulier de l'organisation. Enfin, les auteurs soulignent, après un recensement de la littérature où domine un point de vue fonctionnel du leadership, l'importance de la prise en compte de la dimension émotionnelle du leadership comme phénomène social et contextualisé, inséparable de la fonction plus visible et reconnue du leadership.

4 Le chapitre 2 analyse la nouvelle fonction de directeur d'établissement des écoles primaires dans le canton de Genève, et à partir d'une enquête auprès de 93 directeurs d'établissement, Laetitia Progin explore le concept de leadership pédagogique entre dimension externalisante (régulation de l'apprentissage par un moyen externe comme le soutien scolaire) et internalisante (influence directe sur les pratiques des enseignants). Elle révèle que cette nouvelle fonction est loin d'être clairement circonscrite pour ces directeurs qui présentent des pratiques diverses, à l'image des situations variées et complexes auxquelles ils doivent faire face au quotidien.

5 Cet angle d'analyse est complété par la gestion éducative (chapitre 3) exercée par les directions d'établissement d'enseignement primaire au Québec. Pierre Lapointe et Jean Archambault soulignent les tensions entre deux conceptions du leadership scolaire, l'une traditionnelle, le leadership managérial, et l'autre plus récente, le leadership pédagogique, profil émanant des recherches sur l'efficacité des établissements scolaires dans les années quatre-vingt. L'enquête de terrain auprès de 138 directions montre que celles-ci, en accord avec la littérature, déclarent consacrer autant de temps à la gestion administrative qu'à la gestion éducative dans un contexte d'alourdissement des tâches, conséquence de la montée en autonomie des établissements. Elle révèle par ailleurs un écart important entre les niveaux d'engagement des directions en matière de gestion éducative.

6 La première partie termine sur l'approche du leadership formellement distribué (chapitre 4) dont la fonction s'exerce dans un contexte marqué par des strates multiples, du niveau micro du système (leadership individuel) au niveau macro (les 
hautes-écoles en Fédération Wallonie-Bruxelles et leur environnement, puis les niveaux local, régional et national). En étudiant le travail des directeurs de catégorie dans un paysage en mutation (fusions d'établissements), Caroline Letor et Michèle Garant affinent les contours de la notion du leadership distribué en y apportant des nuances supplémentaires : en plus de la fonction de gestion pure, le leader peut aussi être capable de réflexivité en agissant en situation et en contribuant à l'apprentissage organisationnel. Leur enquête montre que la fonction de leadership s'exerce de manière variable selon les individus : pour certains la démarche est lisible avec des actions coordonnées autour des missions d'enseignement et d'apprentissage, pour d'autres, des difficultés à les relier révélant un traitement isolé des différentes actions. Par ailleurs, si l'espace du micro-système semble investi, il n'en est pas de même des actions liées au macro-système, moins visibles. Enfin, l'enquête permet de dégager trois formes d'exercice du leadership : distribution formelle (verticale par voie hiérarchique ou horizontale entre directeurs), stratégique (pour des missions spécifiques) et culturelle (forme plus spontanée de leadership autour de la dimension culturelle de l'établissement).

7 La deuxième partie de l'ouvrage traite d'un nouveau point d'ancrage dans la ligne hiérarchique du leadership: les cadres de soutien dont la fonction nouvellement dessinée représente une interface interne entre direction et équipe enseignante et servant d'appui au pilotage pédagogique local. Au chapitre 5 , ces cadres intermédiaires, nommés « doyen » dans le canton de Vaud en Suisse, ont pour mission de coordonner et animer l'action pédagogique des équipes d'enseignants. Olivier Perrenoud présente ici le leadership d'équipe comme un acteur capable d'influencer un groupe dans l'accomplissement de missions spécifiques et d'assurer une synergie collective. L'étude met en lumière les attentes et représentations des enseignants de la fonction d'encadrement : un leader de proximité, doté de compétences en animation, gestion d'équipe et communication, et d'une compétence sociale facilitant la gestion individuelle et du groupe.

8 Les cadres de soutien font l'objet dans le chapitre 6 d'un nouvel angle d'analyse. JeanFrançois Marcel aborde la fonction de coordonnateur dans l'enseignement agricole en France dans une perspective de régulation sociale et institutionnelle. En convoquant la théorie de l'analyse stratégique, l'auteur analyse l'espace intermédiaire, à l'intérieur duquel s'élaborent de nouvelles règles du jeu de l'organisation, et qu'occupent trois catégories d'acteurs: équipe de direction, équipe pédagogique et coordonnateur d'équipe. À partir de l'analyse d'un artefact significatif (fiche de poste détaillée) produit par une structure de formation, l'étude montre comment la direction tente d'occuper l'espace à construire en exerçant une forme de contrôle aussi bien sur cette zone d'incertitude que sur le travail du coordonnateur. Quant aux équipes d'enseignants, elles revendiquent un droit de regard sur les modalités de coordination et considèrent légitime leur participation à la construction des règles du jeu organisationnel. Enfin, le coordonnateur constitue lui aussi sa sphère de pouvoir vis-à-vis des deux autres protagonistes en refusant la facette coercitive du rôle attribué par la direction et en assumant une position d'autorité dans ses interactions avec les enseignants. Le résultat final est une appropriation de sa fonction par le coordonnateur à travers une stratégie de mise à distance des concurrents et de coopération avec les enseignants, débouchant sur une reconnaissance de la nouvelle fonction. 
9 En poursuivant la réflexion sur les coordinateurs pédagogiques, Thierry Piot examine leur professionnalité dans un contexte de réingénierie des systèmes d'éducation et de formation autour de la notion de compétences (chapitre 7). Il souligne une différence fondamentale, à notre avis importante pour l'ouvrage, entre les fonctions de direction et de coordinateur, ce dernier étant dépendant dans une grande mesure de l'adhésion du groupe qu'il encadre et de laquelle dépend son influence. L'outil sur lequel s'appuie l'analyse est cette fois le portfolio, lequel est instrumentalisé par les acteurs en vue de composer et structurer leur activité. Multifonctionnel, le portfolio devient aussi un moyen au service de la construction d'une professionnalité naissante tout en contribuant à la régulation et l'individualisation pédagogiques. En convoquant la théorie de la didactique professionnelle, l'auteur analyse, en lien avec l'usage du portfolio, l'activité des coordinateurs pédagogiques des Instituts de formation en soins infirmiers en France, et montre comment ceux-ci détournent l'artefact de sa fonction évaluative primaire pour en faire aussi un outil d'évaluation formative, adapté aux besoins du contexte et au service de la régulation interne. Ainsi, la création de nouveaux schèmes de coordination et de nouvelles images opératives contribuent à la construction de nouvelles compétences en coordination pédagogique.

10 La dernière partie de l'ouvrage aborde les questions de supervision et d'accompagnement à partir d'une démarche de recherche-action au Québec (chapitre 8) dont le but est la production d'un modèle de conduite de l'entretien d'accompagnement pour les directeurs. Louise Royal, Michel Boyer et Martine StGermain révèlent que la mise en place d'un cadre à l'accompagnement pédagogique aide à l'instauration d'une relation moins asymétrique entre directeurs et enseignants, et contribue à des relations de coopération et des comportements réflexifs. L'implication de la recherche met ainsi l'accent sur la régulation des pratiques professionnelles en vue du développement professionnel des accompagnantsaccompagnés.

11 L'ouvrage termine sur une étude comparative (chapitre 9) de l'évolution du métier d'inspecteur dans trois pays (Angleterre, France, Suisse) en lien avec la pratique de l'évaluation externe des établissements scolaires. Elle souligne des tensions et une institutionnalisation contrastée de l'évaluation suivant la place qu'occupent les inspecteurs dans la procédure: entre conception formalisée (full inspection en Angleterre) et conception plus mixte (France, Suisse) laissant plus ou moins de place à l'appréciation personnelle des inspecteurs, entre fonction plus ou moins indépendante ou fonction mise sous tutelle (France), entre compétences de spécialisation et compétences transversales (France et Angleterre). Le métier d'inspecteur est ainsi présenté comme une fonction en pleine mutation.

12 À partir des assises théoriques solides et des outils de recueil de données variés, appliqués à des milieux institutionnels marqués par des différences de fonctionnement, l'ouvrage réussit à cerner les principaux enjeux autour de la question de l'encadrement et du leadership, en alliant des analyses contextuelles et situationnelles à l'action singulière des acteurs dans un environnement hautement complexe que sont les organisations modernes, en prenant en compte la mise en tension de la nouvelle fonction de leader pédagogique, entre compétences de management et compétences d'accompagnement éducatif. 


\section{AUTEURS}

\section{BABY LENHARD}

Enseignante d'anglais, LEGTA Saint-Ismier, Isère 